See discussions, stats, and author profiles for this publication at: https://www.researchgate.net/publication/326598581

\title{
Using Linked Statistical Data to Improve Marine Search and Rescue Operations in Ireland
}

Conference Paper · April 2018

DOI: $10.1145 / 3209415.3209511$

CITATIONS

2

8 authors, including:

Arkadiusz Stasiewicz

National University of Ireland, Galway

9 PUBLICATIONS 55 CITATIONS

SEE PROFILE

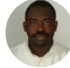

Adegboyega Ojo

National University of Ireland, Galway

165 PUBLICATIONS 1,398 CITATIONS

SEE PROFILE

Some of the authors of this publication are also working on these related projects:

Project ROUTE-TO-PA View project

Project OpenGovIntelligence View project
READS

23

Mohamed Adel

Cairo University

47 PUBLICATIONS 159 CITATIONS

SEE PROFILE

Efthimios Tambouris

University of Macedonia

201 PUBLICATIONS 2,032 CITATIONS

SEE PROFILE 


\section{Using Linked Statistical Data to Improve Marine Search and Rescue Operations in Ireland}

\author{
Arkadiusz Stasiewicz \\ Insight Centre for Data Analytics, \\ Ireland \\ arkadiusz.stasiewicz@insight- \\ centre.org \\ Efthimios Tambouris \\ University of Macedonia and ITI- \\ CERTH, Thessaloniki \\ Greece \\ tambouris@uom.gr
}

National University of Ireland, Galway, National University of Ireland, Galway,

\author{
Mohamed Adel Rezk \\ Insight Centre for Data Analytics, \\ Ireland \\ mohamed.adel@insight-centre.org \\ Evangelos Kalampokis \\ University of Macedonia and ITI- \\ CERTH, Thessaloniki \\ Greece \\ ekal@uom.gr
}

\author{
Adegboyega Ojo \\ Insight Centre for Data Analytics, \\ National University of Ireland, Galway, \\ Ireland \\ adegboyega.ojo@insight-centre.org
}

\author{
Trevor Alcorn \\ Marine Institute, \\ Rinville, Oranmore, Co. Galway \\ Ireland \\ trevor.alcorn@marine.ie
}

\author{
Adam Leadbetter \\ Marine Institute, \\ Rinville, Oranmore, Co. Galway \\ Ireland \\ adam.leadbetter@marine.ie
}

\begin{abstract}
${ }^{1}$
Producing new generation of digital public services from open data is of major interest to policymakers, practitioners and academia in the digital government community. Recent efforts in the area of Linked Statistical Data suggest that the associated multidimensional data cubes are excellent resources that could underpin data-driven digital public services. We describe in this paper a set of tools and approach to exploiting linked statistical data produced from the integration of streams of open marine datasets for developing digital services to support Marine Rescue Operations. We also highlight the opportunities enabled through co-creation activities as well as the benefit and challenges for scaling and sustaining the initiative.
\end{abstract}

\section{CCS CONCEPTS}

.Information systems $\rightarrow$ Information systems applications

\section{KEYWORDS}

Linked Statistical Data, Linked Open Data, Digital Public Service, Open Data, Co-creation, E-Government, OpenGovIntelligence

\footnotetext{
${ }^{1}$ Permission to make digital or hard copies of all or part of this work for personal or classroom use is granted without fee provided that copies are not made or distributed for profit or commercial advantage and that copies bear this notice and the full citation on the first page. Copyrights for components of this work owned by others than ACM must be honored. Abstracting with credit is permitted. To copy otherwise, or republish, to post on servers or to redistribute to lists, requires prior specific permission and/or a fee. Request permissions from Permissions@acm.org.
}

ACM Reference format:

A. Stasiewicz, M. Rezk, A. Ojo, E. Tambouris, E. Kalampokis, K. Tarabanis, T. Alcorn, A. Leadbetter. 2018. Using Linked Statistical Data to Improve Marine Search and Rescue Operations in Ireland. In Proceedings of the $11^{\text {th }}$ International Conference on Theory and Practice of Electronic Governance, Galway, Ireland, April 2018 (ICEGOV'18), 7 pages.

DOI: $10.1145 / 3209415.3209511$

\section{INTRODUCTION}

Open Government Data (OGD) has the potentials to increase government transparency, improve the workings of public administration, contribute to economic growth, and provide social value to citizens (Burdon, 2009, Kucera, 2014). However, the potentials of OGD are far from being fully realised. In this vein, a number of technical, organisational, cultural, and legal challenges remain barriers to be addressed (Janssen et al., 2012). It has been recently suggested that open statistical data provided by National Statistical Institutes and public authorities at various administrative levels can be more easily exploited in the development of value-added services (Kalampokis et al., 2016). This category of data is numerical, well structured, and usually

ICEGOV '18, April 4-6, 2018, Galway, Ireland (C) 2018 Association for Computing Machinery. ACM ISBN 978-1-4503-5421-9/18/04...\$15.00 https://doi.org/10.1145/3209415.3209511 
of high quality. In addition, given the relatively higher information content in statistical data when compared to other categories of data, they provide a compelling basis for digital information services.

However, the development of digital information services based on advanced data analytics services and applications require the integration of data coming from multiple and disparate sources (Kalampokis, 2016). Linked data technologies, which facilitate data integration on the Web, can be employed to represent statistical data. Linked Open Statistical Data (LOSD) have been successfully exploited to support problems in few areas such as public administration (Chaniotaki, 2017), health (McCusker et al., 2013), and biodiversity (Koho et al. 2014). These cases provide initial evidences on how LOSD can be used to solve real-world problems.

In this paper, we present a case on how LOSD could be exploited in the co-creation of digital service for marine search and rescue. Maritime search and rescue operations in Ireland are currently either supported by manually serviced data requests or by daily delivery of data from a RESTful endpoint to a closedsource integrated situational awareness application. We also highlight the expected challenges in meeting the information and decision-making needs of the different actors involved in maritime search and rescue operations.

The rest of this paper is organized as follows. Section 2 presents maritime search and rescue operations in Ireland. Section 3 presents the state-of-play in the processes and tools for exploiting Linked Open Statistical Data in the public sector. Section 4 presents technical requirements and specific usage scenarios in the context of maritime rescue operations, while Section 5 discusses potential benefits and challenges of cocreating LOSD-based digital services. Finally, Section 6 draws some conclusions.

\section{CO-CREATING A MARITIME SEARCH AND RESCUE DIGITAL SERVICE}

In a co-creation and collective intelligence session with local data users including from the local lifeboat station, fire service and a Search and Rescue (SAR) operations, a use case for the exploitation of marine data driven digital service was identified. The session was held on the $3^{\text {rd }}$ of June 2016 with 12 expert marine sector stakeholders participating in the event (see Fig. 1). SAR operations are targeting people who are, or are believed to be, in a situation which is threating to their life. In the use case development session, the coordinator of the search and rescue operation was identified as needing to know past, present and future oceanographic conditions in the waters around the coastline; where teams can access the shorefront; and where an object which has been dropped into the water is likely to have been transported to since it entered the sea. As the use case was developed further the need for team members to be able to return information, such as geo-located photographs, to the coordinator so that they can be kept up to date of the search team's location and conditions was identified. Further, the ability of members of the public involved in searching the coastline to have access to the same apps and a subset of the same data as the authorities was identified as desirable. Finally, the session identified that the team's coordinator review the information collected by the app after each rescue to build up dataset which will inform the development of local search and rescue policies. Such a system was identified as a current gap in the application landscape by those who attended the co-creation session.

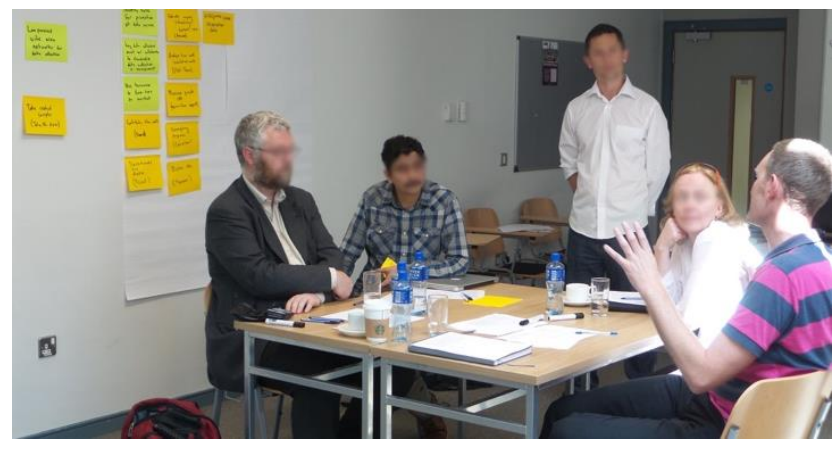

Figure 1: Collective intelligence session held in June 2016

\section{LINKED OPEN STATISTICAL DATA IN THE PUBLIC SECTOR}

Statistical data, including social and demographic indicators, are a valuable resource for the creation of value-added services for businesses and citizens alike. After the emergence of the Open Data movement, statistical data are collected and disseminated not only by National Statistical Institutes but also by various public authorities through Open Data portals at multiple administrative levels (Kalampokis et al., 2016). This has created a new environment where an increased volume of statistical data is freely available for reuse. This new environment, however, is highly fragmented meaning that multiple portals provide data about the same statistical indicator. It is indicative that in the UK, unemployment related statistics can be found in more than 2,000 datasets spread across 18 Open Data portals not including ONS, the UK's National Statistical Institute (Kalampokis et al, 2016).

The exploitation of Open Statistical Data in the creation of value-added data-driven services requires the integration of this fragmented landscape. Towards this end, a number of technical, organisational, social, and legal challenges need to be addressed. From a technological point of view, Linked Data has been introduced as a promising paradigm for opening up data because it facilitates data integration on the Web (Bizer et al., 2011). In the case of statistical data, Linked Data has the potential to realise the vision of performing data analytics on top of integrated but previously isolated statistical data across the Web (Perez et al., 2008). Towards this end, standard vocabularies such as the RDF data cube $(\mathrm{QB})$ vocabulary, SKOS and XKOS enable the creation of Linked Open Statistical Data (LOSD) from raw statistical datasets. 
Using Linked Statistical Data to Improve Marine Search and Rescue Operations in Ireland

However, a number of challenges hamper the creation of LOSD-driven services and applications. Challenges that are related to both publishing and exploitation of LOSD include:

- Although standard Web technologies such as HTTP, $\mathrm{RDF}$ and URIs and standard vocabularies such as RDF data cube, SKOS and XKOS are used for LOSD publishing, different portals often adopt different practices for using these standards. For example, different practices are adopted for the definition of multiple measures and popular dimension (i.e. time, geography) along with their code lists.

- The software tools that enable analysing and visualising LOSD are significantly less mature than the ones focusing on other data formats such as JSON.

- The software tools for exploiting LOSD cannot be reused across different portals.

In the course of the OpenGovIntelligence project we have developed a toolkit for addressing these challenges and enable the wide exploitation of LOSD in the development of added value services.

\subsection{LOSD Publishing}

\subsubsection{Best practices for LOSD publishing}

We have developed a set of best practices for publishing statistical data on the Web following the linked data principles. These practices aim at supporting data publishers to model their data and to apply common linked data standards. In particular, the practices specify:

- How to define a measure and the unit of the measure

- How to define multiple units per measure

- How to define multiple measures

- How to define dimension properties and values

- How to define values of common dimensions such as time and geography

- How to define single value dimensions

- How to define code lists

The wide adoption of such practices can increase interoperability among portals of open statistical data on the Web, and thus facilitate the integration of relevant datasets as well as the development of generic software tools that can be reused across different datasets. The practices are currently online as a working $\operatorname{draft}^{2}$.

\subsubsection{Software tools for LOSD publishing}

The developed tools for LOSD publishing are based on existing open source tools, mainly Grafter. This is a command-line application that enables creating LOSD from various file formats, such as CSV. We have developed two tools that enable creating LOSD from various file formats.

\footnotetext{
2 https://islab-uom.github.io/qbBestPractices/

3 https://github.com/OpenGovIntelligence/json-qb-api-implementation
}

ICEGOV'18, April 2018, Galway, Ireland

Table2qb and Grafter. The Table2qb tool, implemented with Grafter, takes data in a specific tabular structure, either as a CSV or Excel file, and converts it into an RDF Data Cube. Its functionality includes representing the data as a series of observations with dimensions, attributes and measures, and generating the associated Data Structure Definition.

Data Cube Builder. Data Cube Builder is a tool for transforming non-RDF data sources to RDF Data Cube. It is built on top of the pre-existing tool TARQL. Data Cube Builder can be used through multiple interfaces such as desktop UI, command line, web user interface and as a web service.

\subsection{LOSD Exploitation}

\subsubsection{The JSON-QB API}

In order to unleash the full potential of LOSD there is a need to standardize the interaction (i.e. input, output and functionality) with LOSD in a way that facilitates the development of reusable software. The JSON-QB API aims to exploit the advantages of LOSD (e.g. easy data integration) while making data available in a structure and format that is familiar to a larger group of developers. Some of the flexibility, and associated complexity, of linked data is removed, in favour of simplicity and ease of use. Moreover, the API offers a uniform way to access the data, thus enabling the development of generic software tools that can be reused across datasets. The compliant API should follow patterns and practices familiar to "mainstream" web developers, to facilitate the creation of data-driven visualisations and interactive applications. Moreover, it should be suitable for use by a wide range of statistics publishing organisations, so that data users can have a standard interface to LOSD. This will put constraints on the way that publishers manage their data, however, those constraints should be reasonable and manageable.

The architecture of the JSON-QB API is simple and is developed as a middleware between LOSD and the applications that consume the data. The API receives REST calls and translates them to SPARQL queries which are executed at LOSD portals. Then, the returned results are transformed to JSON format that can easily be consumed by applications.

The API was initially implemented as a REST API where a separate parameter was provided for each input ${ }^{3}$. We currently consider to model the input as a JSON object and implement the API based on GraphQL, which is a data query language proposed by Facebook.

\subsubsection{The OLAP Browser}

A typical OLAP browser lets users interactively view and analyze data from different perspectives and with multiple granularities. The developed OLAP Browser capitalises on the Linked Data paradigm and enables enhanced OLAP analytics on top of integrated views of multiple linked statistical datasets. It enables the (a) discovery of datasets on the Web of Linked Data that are structural compatible to integrate, and (b) the creation of 
ICEGOV'18, April 2018, Galway, Ireland

integrated views of multiple datasets. The idea behind the browser is that a user starts with a cube at hand and wants to enrich it with other data on the Web.

OLAP Browser is based on the JSON-QB API for the implementation. The latest release of the OLAP Browser can be found on GitHub4.

\subsubsection{Data Cube Explorer}

The Data Cube Explorer is a web-based tool that catalogues and presents details of available data cubes to the users. It also enables users to preview cube data using pivot table, cube browser and other visualization widgets (see Fig. 2).

The Cube Explorer is responsible for listing, discovering, analysing and visualization of the data from the Data Cubes. It serves as an integrated user interface for the developed platform. The tool is offering customizable statistical analysis and machine learning services using Data Cubes RDF as input data.

In search and rescue operation it could provide data and the visualisation for the last 24 hours before the incident date and the predicted values grouped by longitude and latitude. Example of datasets already explored with this tool include Average Wind Direction, Wave Peak Direction and Height using data sourced from "Irish Weather Buoy Network" and See Temperature using data sourced from "Irish Marine Institute Connemara Model CONN3D".

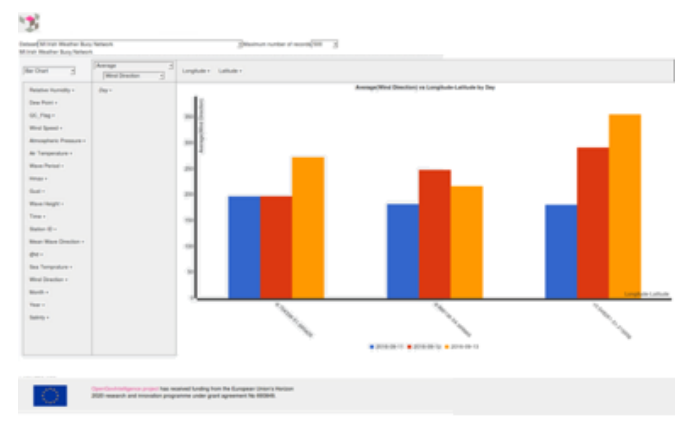

Figure 2: Sample Cube Explorer aggregations.

Cube Explorer users can choose the desired dataset containing the relevant data, choose the visualization and aggregation types and filter out the data to keep only relevant dimensions (e.g. longitude) and measures (e.g. temperature) and the relevant values (e.g. date).

\subsubsection{The Machine Learning Component}

Machine learning enables the creation of predictive models based on the analysis of high volumes of data. The wealth of the statistical data that is freely available online can contribute towards this direction. The multi-dimensional nature of statistical data enables the extraction of numerous features from a single dataset based on the value of the measured variable for the different values of the dimensions or the different aggregated
A. Stasiewicz, M. Rezk, A. Ojo, E. Tambouris, E. Kalampokis, K. Tarabanis, T. Alcorn, A. Leadbetter

functions (e.g. min, max, average etc.) that can be applied to the measure.
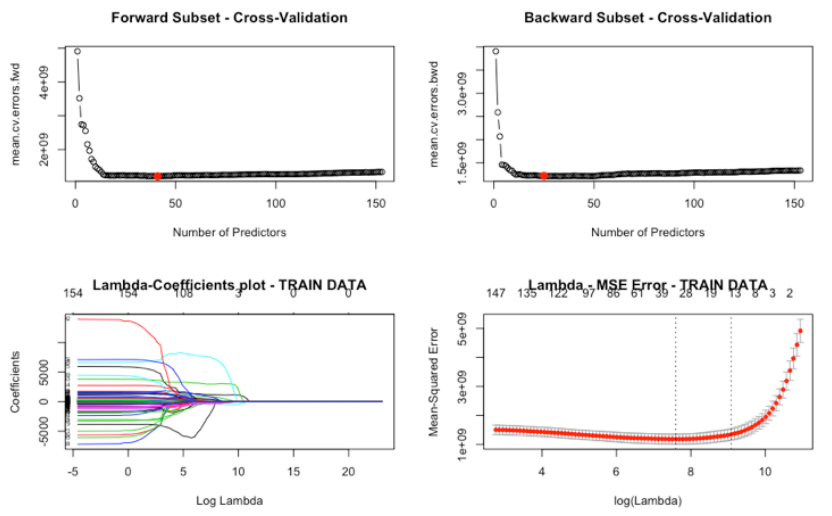

Figure 3: Dimension reduction with the Machine Learning Component

The Machine Learning Component enables the automatic extraction of numerous features from LOSD based on the needs of the users and the predictive scenario that is implemented. It also enables dimension reduction based on relevant algorithms such as Forward Subset, Backward Subset, and Lasso in a user friendly approach. In this way, users will be able to include big volumes of LOSD in machine learning scenarios and filter only the predictors that are relevant to the scenario at hand. These predictors can be later used in the creation of predictive models.

The implementation of the Machine Learning Component is based on the JSON-QB API and R server. An example of the prediction outputs is sown in Fig. 3.

\section{TECHNICAL REQUIREMENTS FOR USING LOSD IN MARITIME OPERATIONS}

While Section 2 described the information needs for maritime search and rescue digital service, this section focuses on technical requirements for using LOSD in meeting these needs.

The Marine Institute (Ireland) is active in oceanography, marine environment, fisheries, technology development, aquaculture, catchment management, ocean energy, and biodiscovery, and it has advanced capabilities in information technology, communications and logistical support. Moreover, it supports the dissemination of data on an open access basis: data from surveys on-board the Research Vessels Celtic Explorer and Celtic Voyager are available on the Marine Institute website. Data collected during the survey are treated in accordance with the MI data policy and significant resource is devoted to quality assure and manage data.

\footnotetext{
${ }^{4}$ https://github.com/OpenGovIntelligence/qb-olap-browser
} 
Using Linked Statistical Data to Improve Marine Search and Rescue Operations in Ireland

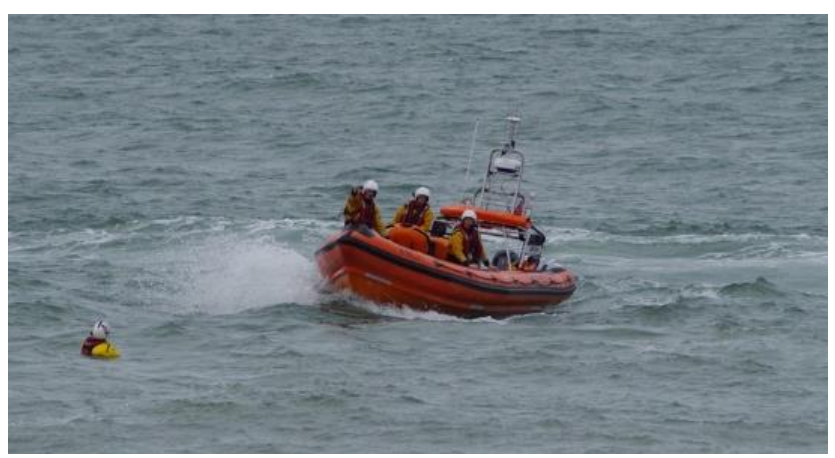

Figure 4: Search and Rescue Lifeboat

Data is available at Marine Institute ERDDAP Server ${ }^{5}$. ERDDAP is a data server that provides collected data in persistent way. The data is arranged in groups and available to download as common machine readable file formats (CSV, JSON, TXT) or as an image: customised graph or map.

At present data published on ERDDAP is used in various data dashboards and online data services such as Irelands Digital Ocean $^{6}$ and Marine Data Online ${ }^{7}$.

Maritime search and rescue operations in Ireland (see Fig. 4) are currently either supported by manually serviced data requests for marine information or by daily delivery of data from a RESTful endpoint to a closed-source integrated situational awareness application.

\subsection{Requirements}

The presented requirements are based on the result of activities performed to identify challenges and needs of Marine Institute. During the stakeholder interviews, Marine Institute described problems that can be solved through the exploitation of statistical data. As a result, we had the opportunity to discuss and document the requirements and design certain scenarios. Table 1. list identified data sources that could support the marine search and rescue operations.

Detailed requirement analysis allowed to specify needs of a tool in which search and rescue personnel can identify the key areas to search for a casualty in the water for rescue or recovery.

Moreover, the expected benefits are defined as follows:

- $\quad$ Reduced recovery search organisation and recovery process

- increased resource allocation effectiveness through LOSD locational intelligence

Table 1: Identified data sources

Dataset Title and Source

\footnotetext{
${ }^{5}$ https://erddap.marine.ie

${ }^{6} \mathrm{https}: / /$ www.digitalocean.ie

${ }^{7}$ http://data.marine.ie

${ }^{8}$ http://jameskinley.tumblr.com/post/37398560534/the-lambda-architecture-principles-for
}

ICEGOV'18, April 2018, Galway, Ireland

1 Latest Sea Surface Temperature (CONN 3D)

https://erddap.marine.ie/erddap/griddap/IMI_CONN_3D.html

2 Latest Sea Surface Height (CONN 2D)

https://erddap.marine.ie/erddap/griddap/IMI_CONN_2D.html

3 Galway Bay Wave Buoy Latest Observations Significant Wave Height https://erddap.marine.ie/erddap/tabledap/IWaveBNetwork_spectral.html

4 Galway Bay Wave Buoy Latest Observations Wave Peak Direction https://erddap.marine.ie/erddap/tabledap/IWaveBNetwork_spectral.html

5 Galway Bay Wave Buoy Latest Observations Mean Wave Period https://erddap.marine.ie/erddap/tabledap/IWaveBNetwork_spectral.html

\subsection{Architecture and LOSD Tools Deployed}

The architecture of the proposed system was based on the lambda architecture pattern ${ }^{8}$ (Fig. 5 and Fig. 6). In general, lambda architecture is used in order to enable implementation of the real-time data processing, which fulfills the needs of maritime search and rescue use case.

Our "maritime search and rescue real-time linked data pipeline" is composed of 10 components: (A.) Digital Oceans Data Service, (B.) Data Acquisition, (C.) Cube Builder, (D.) Cube Models, (E.) Cube Pusher, (F.) Real-Time and Batch Rescue Cube Spaces, (G.) Prediction, (H.) Co-Creation Manager and (I.) Rescue Services.

The components and their interactions are organized as follows:

A. Digital Oceans Data Service: This component serves as a data source for maritime observations. A set of predefined data sets are queried based on the preconfigured time intervals. Batch layer rates are being updated to be used within our pipeline. The identified data sources are listed in Table 1.

B. Data Acquisition: This component is engineered based on the Digital Oceans Data Service API specification. The data is acquired depending on datasets models, speed and batch layer configuration (e.g. update frequency).

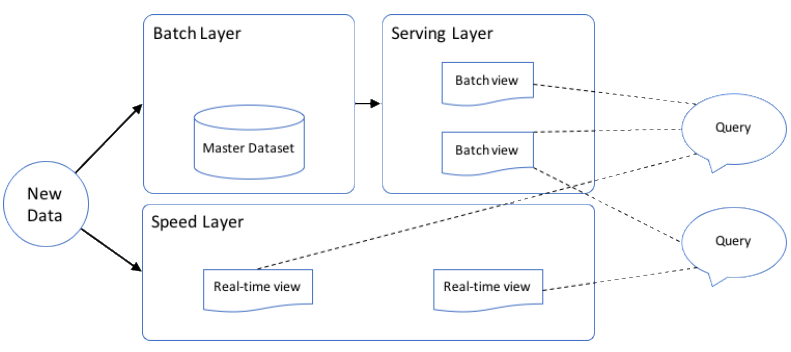

Figure 5: "The Lambda Architectural Framework" 10 


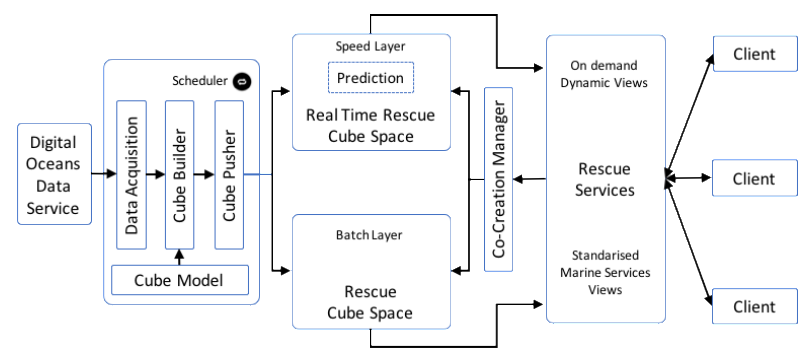

Figure 6: "LOSD-based Maritime search and rescue real time Information Service Architecture"

C. Cube Builder: After data acquisition, it is dispatched to the Linked Data Cube Builder component which use the suitable Data Cube models to transform it into Data Cube.

D. Cube Models: Cube Models are RDF models that describe the mapping details between the legacy data and the RDF Data Cubes. This component is used by the Cube Builder to achieve the data transformation process.

E. Cube Pusher: This component handles the final stage of the Cube Data creation process. The data is being pushed to the RDF data store either through the speed layer or batch Cube Space.

F. Real-Time and Batch Rescue Cube Spaces: This component is realized by the $\mathrm{RDF}$ data store. It receives, stores and serves the created Data Cubes output incrementally. Both Real Time and Batch Rescue Cube Spaces differ in their operations regarding two aspects: updates frequency and storage time window. Update rate for real-time cube space is between $0.5 \mathrm{~h}$ to $1 \mathrm{~h}$ and $24 \mathrm{~h}$ for batch cube space. Time window for real-time cube space is ranged between 3 and 90 days.

G. Prediction: In order to generate the predicted values for the Real Time Cube Space Services' queries, we apply dynamic prediction models over the Real Time Cube Space to generate estimated values for the different time series measures within the data sets. In the current implementation, predicted values are obtained from the predictive models on Marine Institute ERDDAP Server. The Machine learning component described in Section 3.2.4 could also be used.

H. Co-Creation Manager: This component is responsible for clients feedback/inputs management and updating of the Cube Spaces with provided annotations including images. For example, a client might want to link an image, tweet or news article that is related to an observation, data slice, or particular view or a data set. This component will enable updating and merging of the clients inputs with the cube spaces.

I. Rescue Services: This layer of the architecture provides two types of maritime rescue information services dynamic queries on past, current and future measurements (real-time views) and the standardised
A. Stasiewicz, M. Rezk, A. Ojo, E. Tambouris, E. Kalampokis, K. Tarabanis, T. Alcorn, A. Leadbetter

views. These layers will be implemented using OLAP Browser and Cube Explorer described in Sections 3.2.2 and 3.2.3 respectively.

\section{BENEFITS AND CHALLENGES}

In this section, we first describe some of the benefits that could accrue from the adoption and use of the described LOSD- based solution for marine rescue service. First, the use of the LOSD approach enabled the transformation of relevant datasets from the native maritime data formats (e.g. SeaDataNet formats) to linked open data, making it possible to integrate these datasets with structured and unstructured data from other sources.

Secondly, as indicated in Section 2, there is currently no realtime on-demand maritime rescue information service. It is plausible to assume that access to dynamic and real-time information to support rescue operations will positively impact rescue outcomes.

Thirdly, the availability of technical infrastructure of the nature presented in this paper should positively impact the public value regarding 1) maritime search and rescue operations, 2) public sector innovation in the area of marine data-driven services and 3) co-creation between marine data providers and marine data users in the maritime search and rescue sector.

However, a number of challenges may arise once beyond the pilot phase of the proposed solution for a number of reasons. The solution clearly introduces additional operational costs and overheads that could either be borne by the service provider or shared between the service provider and users. There are also potential legal and ethical issues associated the use of the information services, particularly in the area of predicted values or contributed artefacts by third parties. Mishaps could result from inaccurate predictions of oceanographic measurements or problems with artefacts contributed by members of public as part of the co-creation process. These challenges and others are planned to be addressed with contributions from the different stakeholders.

\section{CONCLUSIONS}

The target users of presented service are members of the search and rescue teams. The work described here is a step towards improvements in outcomes for search, rescue and recovery operations. In future, we plan to identify more specific services that will support users in the identification of the key areas to search in the event of a casualty in the water for both rescue and recovery operations. From the technical perspective, we shall seek to refine the tools described in Sections 3 targeted specifically at supporting rescue as well as other maritime activities. Future efforts will also include further investigations of the values and benefits of the use of Linked Open Statistical Data and overcoming the challenges encountered so far during the OpenGovIntelligence project. 
Using Linked Statistical Data to Improve Marine Search and Rescue Operations in Ireland

\section{ACKNOWLEDGMENTS}

The work reported this paper is funded by the EC within the H2020 Program in the context of the OpenGovIntelligence project under grand agreement No. 693849.

\section{REFERENCES}

[1] Bizer, C., Heath, T., \& Berners-Lee, T. (2009). Linked Data - The Story So Far. International Journal on Semantic Web and Information Systems, 5, 122. http://doi.org/10.4018/jswis.2009081901

[2] Burdon, M. (2009). Commercializing Public Sector Information. IEEE Technology \& Society Magazine, 28(1), 34-40. http://doi.org/10.1109/MTS.2009.931860

[3] Chaniotaki, E. (n.d.). Exploiting Linked Statistical Data in Public Administration: The Case of the Greek Ministry of Administrative Reconstruction, 1-5.

[4] Kalampokis, E., Tambouris, E., Karamanou, A., \& Tarabanis, K. (2016). Open statistics: The rise of a New Era for open data? In Lecture Notes in Computer Science (including subseries Lecture Notes in Artificial Intelligence and Lecture Notes in Bioinformatics) (Vol. 9820, pp. 31-43). http://doi.org/10.1007/978-3-319-44421-5_3

[5] Kalampokis, E., Tambouris, E., \& Tarabanis, K. (2016). Linked Open Cube Analytics Systems: Potential and Challenges. IEEE Intelligent Systems, 31(5), 89-92. http://doi.org/10.1109/MIS.2016.82

[6] Kalampokis, E., \& Tarabanis, K. (n.d.). Visualizing Linked Open Statistical Data to Support Public Administration.

[7] Koho, M., \& Hyv, E. (2014). The Semantic Web: ESWC 2014 Satellite Events, 8798, 75-85. http://doi.org/10.1007/978-3-319-11955-7

[8] Kucera, J., \& Chlapek, D. (2014). Benefits and Risks of Open Government Data. Journal of Systems Integration, 30-41. http://doi.org/10.20470/jsi.v5i1.185

[9] Leadbetter, A., Arko, R., Chandler, C., Shepherd, A., \& Lowry, R. (2013) Linked Data. An Oceanographic Perspective, 7-13.

[10 McCusker, J. P., McGuinness, D. L., Lee, J., Thomas, C., Courtney, P.,

Tatalovich, Z., ... Shaikh, A. (2013). Towards next generation health data exploration: A data cube-based investigation into population statistics for tobacco. Proceedings of the Annual Hawaii International Conference on System Sciences, 2725-2732. http://doi.org/10.1109/HICSS.2013.567

[11 Pérez, J. M., Llavori, R. B., Aramburu, M. J., \& Pedersen, T. B. (2008).

] Integrating Data Warehouses with Web Data: A Survey. IEEE Trans. Knowl Data Eng., 20(7), 940-955.

[12 Toots, M., Mcbride, K., Kalvet, T., Krimmer, R., Tambouris, E., Panopoulou,

E., Kalampokis E., Tarabanis, K. (2017). A Framework for Data-Driven Public Service, 264-275. http://doi.org/10.1007/978-3-319-64677-0 